\title{
Design Optimization of a High Speed Synchronous Reluctance Machine
}

\author{
M. Di Nardo, G. Lo Calzo, M. Galea, C. Gerada
}

\begin{abstract}
Synchronous reluctance machines, including the permanent magnet assisted variants are competitive motor topologies if the application requires high efficiency and a cost effective solution with a high flux weakening capability. However, increasing operating speeds incur challenging design and development decisions, mainly in order to find design solutions that ensure the machines structural integrity without compromising the overall performance. In this paper, a comprehensive design procedure for high speed synchronous reluctance machines is presented. In order to validate the procedure, a $5 \mathrm{~kW}, 80000 \mathrm{rpm}$ machine is considered. The proposed strategy consists of a two-step procedure in which the electromagnetic and structural designs have been properly decoupled dividing the design space in two subsets. Each subset mainly affects the electromagnetic or the structural performances. Several structural design optimizations have been then performed with the aim of finding the optimal trade-off between the rotor geometrical complexity (that defines the required computational resources) and the electromagnetic performance. The reported experimental tests of the prototyped machine validate the proposed design strategy which can be used as general guidelines on the structural design of synchronous reluctance machines.
\end{abstract}

Index Terms-Finite element analysis, high speed, multiphysics design, multi-objective optimization, rotor structural design, synchronous reluctance machines.

\section{INTRODUCTION}

$\mathbf{S}$ ynchronous reluctance (SyR) machines are currently experiencing a revamped industrial interest mainly because when compared to the more common induction machines (IM), they can achieve considerable volume reduction and/or an improvement in operational efficiency [1]. Along with the higher efficiency, high transient overload capability [2], simple rotor construction and no need of rare earth permanent magnets (PMs) are the other main advantages of SyR machines which make them a low cost alternative. On the other hand, high torque ripple, low power factor and a slightly reduced flux weakening range (compared to an IM drive having the same converter kVA rating) are its main disadvantages. The transversal laminated SyR machine with multiple flux barriers has been extensively studied since the 1990s [3]. In particular considerable research has been focused on the main design challenges of SyR machines, namely torque ripple minimization and the maximization of the saliency ratio [4]. A number of different techniques for torque ripple minimisation have been developed, including rotor skewing, an appropriate choice of the airgap positions and the number of flux barriers with respect to the number of stator slots [5]. Other techniques address optimization of the flux barrier

M. Di Nardo, G. Lo Calzo, M. Galea, C. Gerada are with Power Electronics and Machine Control Group, University of Nottingham

(email: mauro.dinardo@nottingham.ac.uk) and end-barrier geometries [6]. Considerable improvements especially related to the power factor and the constant power speed range can be achieved by the PM-assisted variant (PMaSyR) where low energy density PMs are inserted in the rotor flux barriers [7]. Although these machine topologies can be considered a valuable alternative to the more common IM and PM synchronous machines (PMSM) in the low-medium speed range, as the speed increases the conflict requirements between electromagnetic torque and structural integrity can potentially compromise its overall performance. In fact, the rotor integrity is practically dependent on the so-called iron bridge (also known as ribs or posts), which can be observed in Fig. 1. If these bridges are made to be wider for the sake of the rotor robustness at high speed, then this comes at the cost of a reduction in torque and power factor. Traditionally, all the above has significantly limited the use of SyR and PMaSyR machines for applications operating in medium to high speed ranges. Mainly four design approaches have been recently presented aimed at overcoming the structural limitations of SyR and PMaSyR when applied in high speed applications. The first makes use of solid rotor structure [8], the second encompasses the use of external rotor sleeve [9], the third one adopts a new dual-state soft magnetic material [10] while the last one involves the use of high strength soft magnetic material [11]-[13]. Solid SyR rotor structures could require particular manufacturing process and the machine performances (especially in terms of efficiency) are deeply limited by the high rotor losses. Adding an external sleeve to a laminated SyR rotor definitely relieves the mechanical stress experienced by the iron bridges but inevitably involves the increment of the physical air-gap and complicates the rotor manufacturing. The use of the advanced, complex materials such as the dualstate material (i.e. a material whose permeability can be locally reduced via heat treatments) gives significant advantages over

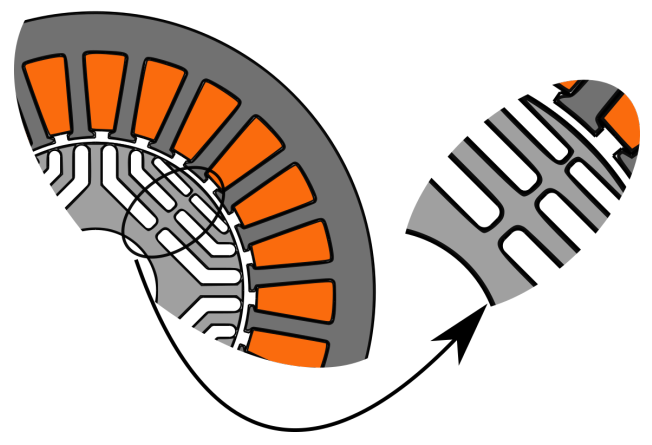

Fig. 1: Cross sectional sketch of a SyR machine with zoom on the central iron bridges 
all the structural speed-dependent limitations; however this material is not an off-the-shelf product indicating high costs and low availability. The last design approach comprises the adoption of improved electrical steel materials that while still "conventional" are specifically designed to meet the higher demand in yield strength with minimum effect on the electromagnetic characteristics. In this work, the last approach has been adopted since it makes use of the best available off-the-shelf materials and it does not require any particular manufacturing process.

\section{High SPEED SyR DESIGN CHALLENGES}

When compared to other high speed machine topologies, mainly PMSM, IMs and switched reluctance (SR) machines, the high speed SyR machine (HS-SyR) has the following benefits. They do not require any rotor retaining system which make the rotor construction more complex and increase the rotor losses, they reduce the risk of uncontrolled voltage operation in case of inverter fault and they still exhibit high efficiency and torque density without the need of using rare earth PMs. Despite these advantages, particular care must still be devoted to the rotor design of HS-SyR machines since structural and electromagnetic aspects have to be considered with equal importance. In order to achieve good HS-SyR designs, able to satisfy the electromagnetic performance and rotor integrity requirements, several multi-physics design approaches have been proposed [13]-[15]. It has been shown [14] that, if a FE-based optimization is adopted as design approach, then dividing the design procedure in two steps, i.e. the electromagnetic design followed by a proper mechanical design, is beneficial for both points of view of the computational effort and performance of the final solution. Considering that the electromagnetic design and analysis of SyR machines had been extensively covered in other works, then this paper is mainly focused on the structural design aspects. Several attempts [16], [17] have been made in order to identify guidelines and analytical rules to design the iron bridge dimensions. The most common [16] is able to estimate the total iron bridge per flux barrier; however this strongly depends on the rotor geometry under consideration (end barrier geometry, fillet radii of the barrier corners, etc.). This analytical formulation therefore necessitates the use of safety factors that have to be preliminary identified by finite element (FE) analysis or based on the designer's experience. In addition, such analytical model cannot predict the optimal spatial distribution of the estimated total bridge, i.e. the subdivision between the tangential and radial ribs and the positions of the latter along the flux barrier. The aforementioned model is thus usually used to initially estimate the total iron bridge dimensions, however a structural FE design stage is always needed to verify the initial estimated guess and individuate its optimal spatial redistribution [13]. Regarding the latter, in [15] it has been shown that the position of the radial ribs plays a key role in the minimization of the maximum von Mises stress. However such results are obtained via a parametric study which obviously does not take into account the interaction of all rotor parameters on the structural performances. Considering all the above, it can be deduced that the major issues in the rotor structural design of HS-SyR machines are the high number of geometrical degrees of freedom $(G D O F)$ and the lack of an accurate analytical model. Thus, in this work a stochastic optimization algorithm (OA) coupled with a structural FE is used to carry out the mechanical design of a $5 \mathrm{~kW}-80000 \mathrm{rpm}$ synchronous reluctance machine. It is important to note that FE-based design by means of OA has the serious drawback of considerable computational burden. This is due to 1) the long FE simulation time needed to evaluate a single rotor candidate and 2) the high number of FE evaluations required by the OA to converge. The first is related with the trade-off between required accuracy (which mainly depends on the FE mesh setting) and to the single FE simulation time. The second depends on the selected OA, the number and type of objective functions and the number of input variables (i.e. geometrical complexity) which defines the research space dimension. In particular the latter constitutes a crucial aspect, therefore this paper deeply investigates the compromise between geometrical complexity, obtainable performance and computational time for a given $\mathrm{OA}$ and optimization goal. In the next section the preliminary design choices, the rotor parametrization, the design procedure along with the electromagnetic and structural objectives are presented. Section IV outlines the results of the structural design optimizations both neglecting and considering the influence of the rotor-shaft coupling on the mechanical designs. In section $\mathrm{V}$, the performance of the optimal design are FE-analysed in detail while section VI briefly describes the experimental set-up and presents the comparison between the measured and the expected performance.

\section{Problem Statement}

\section{A. Specifications and preliminary design considerations}

The external dimensions of the HS-SyR machine have been imposed by the constraints of the specific application summarized in Table I along with the other main geometrical parameters. The airgap thickness is fixed to the minimum allowed by the bearing manufacturer being the average torque and power factor of a SyR machine monotonically decreasing as the airgap length increases [18]. A distributed winding configuration is chosen to limit the harmonic content of the stator magneto motive force which negatively influences the iron losses and the reluctance torque [19]. The number of poles is set to 4 with the aim of limiting the fundamental frequency. Considering that 1) a higher number of flux barriers improves magnetic performance but increases construction complexity and reduces overall robustness and 2) for a good trade-off

TABLE I: Machine specifications and main dimensions

\begin{tabular}{ccc}
\hline Parameter & Value & Unit \\
\hline Rated power & 5 & $\mathrm{~kW}$ \\
Maximum speed & 80000 & $\mathrm{rpm}$ \\
Stator diameter & 60 & $\mathrm{~mm}$ \\
Split ratio & 0.54 & - \\
Stack length & 30 & $\mathrm{~mm}$ \\
Airgap length & 0.3 & $\mathrm{~mm}$ \\
Cooling type & Water cooled & - \\
\hline
\end{tabular}


between torque ripple and iron losses, the number of flux barriers is strictly related with the number of stator slots, then a stator with 24 slots and a rotor having three flux barriers per pole is chosen. The choice of the soft magnetic material was based on the different requirements of the stator and the rotor. Thus an optimal material was individually selected for the stator and rotor. The stator is realized using an electrical steel with low specific iron losses $(10 \mathrm{~W} / \mathrm{kg}$ at $1 \mathrm{~T} / 400 \mathrm{~Hz})$ while for the rotor a high strength lamination material has been adopted. The latter has a yield point of $822 \mathrm{MPa}$ at the expense of an increased specific iron loss $(45 \mathrm{~W} / \mathrm{kg}$ at $1 \mathrm{~T} / 400 \mathrm{~Hz})$. Starting from the above constraints and choices and considering a rated torque of $0.6[\mathrm{Nm}]$ and a rated current density of $20\left[\mathrm{~A} / \mathrm{mm}^{2}\right]$, the main stator parameters have been analytically determined [18]. A lumped parameter thermal network is then employed in order to verify the thermal limits of the machine. Considering that the main aim of this work is the rotor design, then the stator is assumed fixed for the rest of the paper.

\section{B. Rotor parametrization}

Different rotor flux barrier parametrizations have been presented in literature [20], [21]. All of them show that the flux barrier and end barrier geometry play a key role on the torque maximization and torque ripple reduction [22]. In general, it has been shown that slightly improved performances can be achieved at the cost of a more complex design (increased number of rotor parameters) of the barrier profiles Thus if a FE-based design assisted by OA is chosen, then the computational burden increases [21]. On the other hand, if an analytical design followed by a FE refinement stage is carried out, the FE stage will be greatly complicated and lengthened even if the most accurate analytical model is implemented [23], [24]. In fact, the latter always require a set of assumptions such as the simplification of the rotor geometry and neglecting the iron non-linearity (also in terms of local saturations). Considering all the above, a rotor geometry described by a reduced number of parameters has been selected and this is shown in Fig. 2a. Each flux barrier is made of straight segments and is unequivocally identified by the radial thickness $h_{i}$, the angular position at the airgap $\Delta \alpha_{i}$ and a set of iron bridges which have to guarantee the structural integrity at high speed. In particular, except for the most external barrier, all of them include tangential, central and lateral iron ribs $T B_{i}, C B_{i}$ and $L B_{i}$, and the latter can assume whatever geometrically feasible

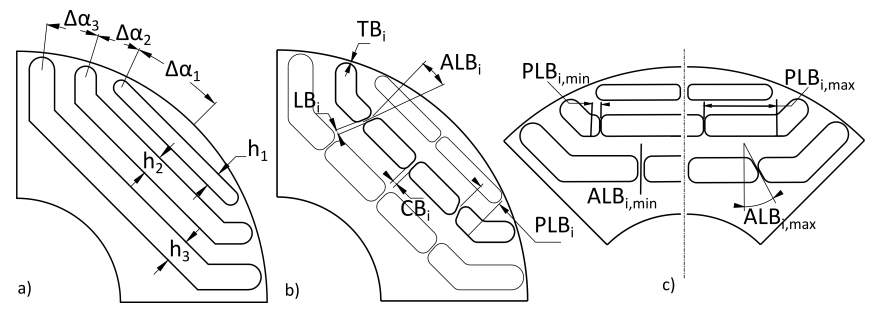

Fig. 2: a) Parametrization of the main part of the flux barriers, b) Parametrization of the iron bridges, i.e. thickness's, position and bending angle of the lateral rib for one flux barrier, c) minimum and maximum $P L B_{i}$ and $A L B_{i}$ position $P L B_{i}$ and bending angle $A L B_{i}$ along the straight part of the barrier (Fig. 2b). Each corner created by the radial posts is rounded with a radius equal to one third of the height of the respective barrier.

\section{Design procedure}

The adopted rotor design method of the HS-SyR machine under study consists of a two-step procedure where an electromagnetic design is followed by a proper mechanical design. This strategy is based on the assumption that the design variables can be divided in two subsets, and each of them is separately sequentially identified.

- The first subset contains 6 geometrical parameters, shown in Fig. 2a, mainly affecting the electromagnetic performances, i.e. radial height $h_{i}$ and angular position $\Delta \alpha_{i}$ of each barrier.

- The second includes 12 variables, shown in Fig. 2b, primarily influencing the von Mises stress distribution of the rotor, i.e. all the iron bridge dimensions $\left(T B_{i}, C B_{i}\right.$ and $\left.L B_{i}\right)$, and the position and bending angle of the lateral rib $\left(P L B_{i}\right.$ and $A L B_{i}$ ).

The hypothesis behind this subdivision is the weak impact of the first subset of parameters on the structural performance and of the second subset on the electromagnetic ones. The above procedure is valid only when the considered structural design criterion directly or indirectly leads to the minimization of the iron bridge thicknesses. This design concept has already been validated in [14], where it has also been shown that, if a FE based design assisted by OA both for structural and electromagnetic aspects is adopted, a substantial reduction of the computational time is achieved.

\section{Electromagnetic and structural performance indexes}

Regarding the electromagnetic design step, two design approaches can be implemented. The first one analytically estimates the main rotor geometrical parameters and makes use of a FEA as refinement stage to take into account the nonlinearities and the disregarded aspect of the analytical model. The second one is a FE-based design assisted by OA. In both cases, average torque and torque ripple are the most important performance indexes to consider during the rotor design. In this case study, the second approach has been adopted and more details can be found in [14].

From a structural design perspective, the lack of an accurate mechanical analytical model and the high number of geometrical degrees of freedom involved in the rotor design has led to the adoption of structural FE-based assisted by OA design approach [25]. The rotor structural integrity at the maximum speed is obviously guaranteed if the maximum von Mises stress experienced in the rotor lies below the yield strength of the lamination material. Consequently during the optimization if the maximum stress exceeds the limit, the rotor candidate has to be penalized. However several combinations of the iron bridge dimensions and distributions can assure the previous essential safety condition, therefore another criterion has to be identified. A possible solution is reported in [14], where the rotor is designed maximizing the mechanical exploitation 
of the iron bridges; such criterion is fulfilled if the same maximum stress is measured in each structural rib. By doing so, the thicknesses of the iron bridges are implicitly minimized leading to highest torque possible for a given stress limit. The major drawback of such structural criteria lies on the difficulty of evaluating this objective function and on its high nonlinearity which prevents the OA to achieve repeatable results. Another possible structural design criterion can be represented by the minimization of the average total bridge $(A T B)$ per flux barrier, defined as:

$$
A T B=\frac{1}{n} \sum_{i=1}^{n}\left(2 \cdot T B_{i}+2 \cdot L B_{i}+C B_{i}\right)
$$

where $\mathrm{n}$ is the number of flux barrier ( 3 in our case study). The application of such criteria lies on the hypothesis that the decrement of the average torque $d T$ caused by the increment of an iron bridge thickness $d B$ is independent from the real position of the rib's increment. In other words, wherever $d B$ is allocated, such as on the tangential, lateral or central rib and whatever the position of the lateral one is, the loss of the torque $d T$ remains the same. As verified later, the minimization of (1) subject to the constraint on the maximum stress (FE calculated), which have to be lower than $657 \mathrm{MPa}$ (i.e. $80 \%$ of the yield strength, $822 \mathrm{MPa}$ ), will lead to rotor structures with the highest average torque for a given stress limit.

\section{Structural DESIGN OPTIMIZATIONS}

In this work a differential evolution optimization algorithm has been selected to perform the HS-SyR rotor structural design. This choice comes from a preliminary optimization campaign in which the convergence time and repeatability of the results of the most common stochastic OAs have been compared. This algorithm definitely gives the best results for the objective problem under consideration. The boundaries of the research space are shown in Table II. The lower boundaries of the radial ribs thicknesses $\left(L B_{i}, C B_{i}\right)$ are zero, however they are not inserted in the corresponding barrier if their thickness, generated by the $\mathrm{OA}$, is lower than the minimum iron thickness $(0.1 \mathrm{~mm})$. By doing so, only rotor structures that can be easily manufactured are considered during the optimization. In order to evaluate the trade-off between obtainable performance and number of geometrical variables and thus computational time, several rotor geometries showing different degrees of freedom $(G D O F)$ in terms of iron bridge thicknesses and distributions have been optimized. Five rotors having an iron bridge parametrization described by an increasing number of variables have been optimized obviously keeping the airgap angular position and heights of the flux barriers equal to the optimal values obtained with the electromagnetic optimization. In particular, each parametrization is described in the following:

- $4 G D O F$ has equal tangential bridge thicknesses $T B$, different central ribs $C B_{i}$ and do not present the lateral posts $L B_{i}$ for the second and third barriers;

- $6 G D O F$ features equal tangential bridge thicknesses $T B$, different radial ribs $C B_{i}, L B_{i}$ but the position $P L B_{i}$ and the bending angle $A L B_{i}$ of $L B_{i}$ are set to zero;
TABLE II: Boundaries of the research space

\begin{tabular}{cccc}
\hline Parameter & Lower & Upper & Unit \\
\hline$T B_{1,2,3}$ & 0.1 & 0.7 & $\mathrm{~mm}$ \\
$C B_{1,2,3}$ & 0 & 0.7 & $\mathrm{~mm}$ \\
$L B_{2,3}$ & 0 & 0.7 & $\mathrm{~mm}$ \\
$P L B_{2,3}$ & 0 & 1 & p.u. \\
$A L B_{2,3}$ & 0 & 30 & deg. \\
\hline
\end{tabular}

- $8 G D O F$ can assume whatever value inside the boundaries of the tangential, central and lateral bridges $T B_{i}, C B_{i}, L B_{i}$, but the variables $P L B_{i}$ and $A L B_{i}$ are still set to zero;

- $10 G D O F$ respect to the previous, has the further possibility of varying the positions of the lateral posts $P L B_{i}$ inside the defined bounds;

- 12 GDOF can additionally present a bending angle of the lateral rib $A L B_{i}$ different than zero.

Each rotor structure has been optimized carrying out 10 optimization runs in order to evaluate the repeatability of the results. As the $G D O F$ increases, the number of performed functional evaluations has been incremented allowing the algorithm to converge as it will be discussed in the next paragraph. In order to achieve the most accurate result in a reasonable amount of time, the FE settings have been customized with the aim to refine the mesh only in the critical parts of the rotor, i.e. the iron bridge regions. Adopting these settings, the FE evaluation of the von Mises stress distribution needed to extrapolate the maximum stress and the computation of the objective function $A T B$, requires 8 seconds per rotor (on an Intel Core i7, $3.6 \mathrm{GHz}, 16 \mathrm{~GB}$ RAM).

\section{A. Optimization results}

The average total bridge evolutions during the optimization of all rotor structures are shown in Fig. 3. For each considered rotor geometry, the best and the worst runs have been reported. Analysing the optimization evolutions, the following considerations regarding the trade-off between obtainable performance and required functional evaluations can be drawn.

- When moving from a 4 to $6 G D O F$ rotor, it is always possible to achieve a substantial reduction (35-40\%) of the $A T B$ even with the same functional evaluations. Therefore splitting the radial ribs into three, one placed in the middle and two at the extreme ends of the straight part of the barrier allows obtaining a better performance index.

- Comparing 6 with $8 G D O F$ rotors, then to obtain a reduction of $5 \%$ or less of the optimization goal, the functional evaluations have to be almost doubled (from 5400 to 10000). Consequently it can be stated that considering tangential ribs with different thicknesses can lead to a weak improvement of the ATB at the cost of remarkable increase of the functional evaluations and so of the computational time.

- Going from 8 to 10 GDOF rotors, then to assure an additional improvement of $5 \%$ (in the best case), the evaluation calls have to be at least increased by $35 \%$; this small improvement can be achieved optimizing also the positions of the lateral ribs.

- A further increment $(35 \%)$ of the number of functional evaluations, going from 10 to 12 GDOF rotors will not 

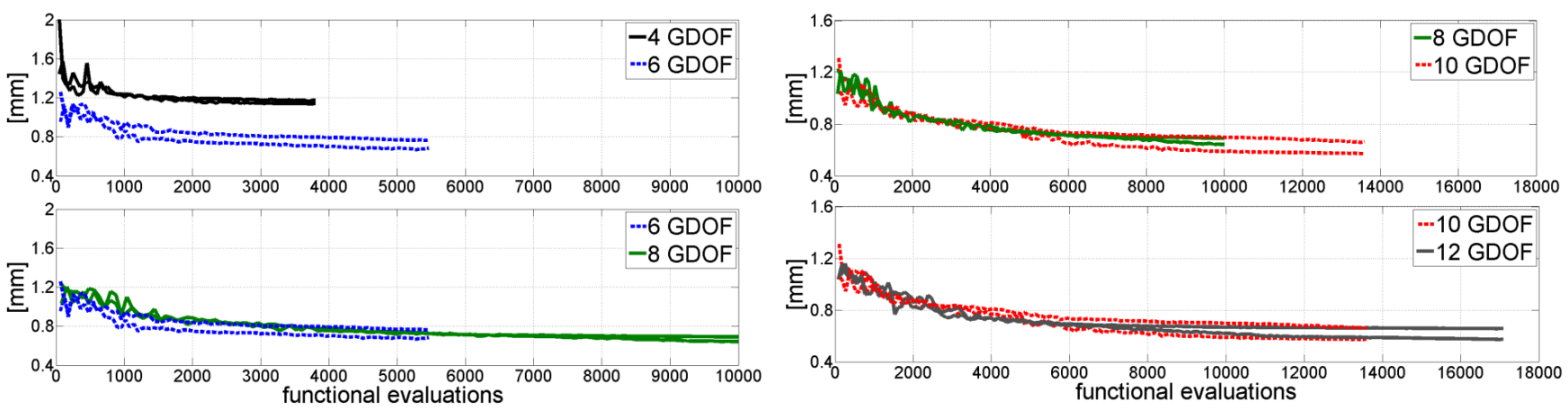

Fig. 3: Average total bridge evolutions for the 5 considered rotor geometries

result in any improvement. It can be then concluded that optimizing the bending angles of the lateral ribs do not lead to any benefit.

It can also be argued that the optimizations of the rotor having 12 GDOF could achieve better performance if the number of functional evaluations is increased. However, it has to be considered that one optimization run (of the 12 GDOF rotor) shown here has been obtained after 38 hours of calculations and the increment of the functional evaluations would have made the computational time impracticable.

\section{B. Analysis of the optimized rotor geometries}

The improvements of the mean torque $\Delta T$ (calculated at $4 / 3^{r d}$ the rated current) caused by the reduction of the average total bridge $\triangle A T B$ respect to the simplest rotor as function of the $G D O F$ are shown in Fig. 4. The final results of the best and worst optimization runs are reported. Considering the best case scenario for each $G D O F$, it can be deduced that passing from rotor geometries comprising:

- 4 to $6 G D O F$, then the $41 \%$ reduction of $A T B$ causes the increment of the mean torque of about $8.6 \%$;

- 6 to $8 G D O F$, then the additional $5.1 \% A T B$ decrement leads to another $1.5 \%$ torque improvement;

- 8 to $10 G D O F$, then the extra $9.8 \%$ decrement of the $A T B$ induces the production of $1.8 \%$ more torque;

- 10 to $12 G D O F$, then there is no change in $A T B$ and therefore in the mean torque.

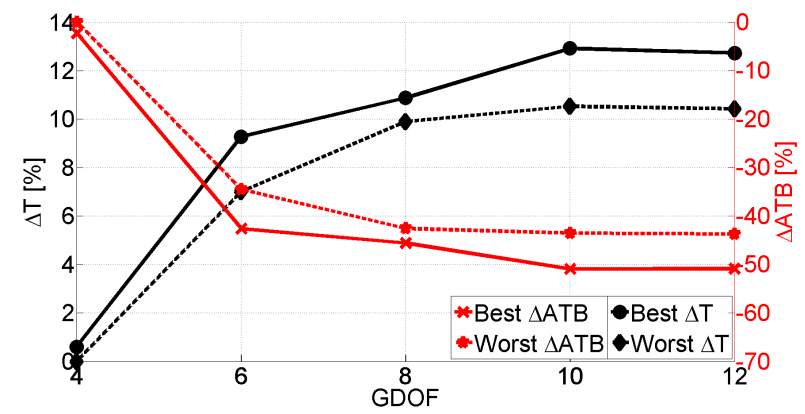

Fig. 4: Average total bridge and mean torque as function of the $G D O F$ for the best (continuous line) and worst (dashed line) optimization runs
Table III reports the iron bridge dimensions and the electromagnetic performances for the optimal rotors of each best optimization run while Fig. 5 show their von Mises stress distributions. It is worth to underline that passing from rotor with 6 to 8,10 or $12 G D O F$, the number of radial bridges $(N R B)$ decreases from 7 to 5 . In particular, comparing the rotor with 6 and $8 G D O F$, the latter does not have the lateral ribs on the second barrier but it has a bigger tangential bridge $T B_{2}$; furthermore the dimension of $L B_{3}$ is remarkably reduced. These changes simplify the rotor construction, especially in case of small rotor bore diameter, because it is preferable to have a reduce number of iron bridges with a larger thickness than a higher number of thinner ribs. However this advantage comes at the price of an increased torque ripple with respect to the initial machine. In fact the torque ripple of the machine without any radial bridges (and all $T B_{i}$ equal to $0.3 \mathrm{~mm}$ ), which is the output of the electromagnetic design stage, is $1.14 \%$ (in standard deviation, std). More the tangential bridge thicknesses vary from the initial value $(0.3$ $\mathrm{mm}$ ), and/or more they differ respect to each other, more the torque ripple changes, as it is for the rotors with 8 and 12 GDOF (see Table III). Differently from the other geometries, the rotors with 8 and 12 GDOF do not have tangential bridge thicknesses which increase from the outermost to the innermost barrier. All the optimized rotor present increasing central and lateral rib widths going from the outermost to the inner most barrier. Analysing the geometry with $10 \mathrm{GDOF}$, it has to be highlighted that the optimal positions of the

TABLE III: Geometrical parameters and electromagnetic performance of the optimal rotors

\begin{tabular}{ccccccc}
\hline & 4 GDOF & 6 GDOF & 8 GDOF & 10 GDOF & 12 GDOF & Unit \\
\hline$T B_{1}$ & 0.48 & 0.11 & 0.12 & 0.11 & 0.11 & $\mathrm{~mm}$ \\
$T B_{2}$ & $=$ & $=$ & 0.22 & 0.10 & 0.20 & $\mathrm{~mm}$ \\
$T B_{3}$ & $=$ & $=$ & 0.12 & 0.15 & 0.13 & $\mathrm{~mm}$ \\
$C B_{1}$ & 0.10 & 0.12 & 0.12 & 0.18 & 0.13 & $\mathrm{~mm}$ \\
$C B_{2}$ & 0.14 & 0.13 & 0.19 & 0 & 0.22 & $\mathrm{~mm}$ \\
$C B_{3}$ & 0.29 & 0.25 & 0.28 & 0 & 0.25 & $\mathrm{~mm}$ \\
$L B_{2}$ & - & 0.11 & 0 & 0.15 & 0 & $\mathrm{~mm}$ \\
$L B_{3}$ & - & 0.33 & 0.20 & 0.26 & 0.12 & $\mathrm{~mm}$ \\
$P L B_{2}$ & - & - & - & 0.52 & 0.43 & p.u. \\
$P L B_{3}$ & - & - & - & 0.65 & 0.08 & p.u. \\
$A L B_{2}$ & - & - & - & - & 11.6 & deg. \\
$A L B_{3}$ & - & - & - & - & 5.2 & deg. \\
\hline$A T B$ & 1.14 & 0.67 & 0.63 & 0.57 & 0.57 & $\mathrm{~mm}$ \\
$N R B$ & 3 & 7 & 5 & 5 & 5 & - \\
\hline$T_{m}$ & 0.739 & 0.802 & 0.815 & 0.829 & 0.828 & $\mathrm{Nm}$ \\
$T_{\text {rip }}$ & 1.67 & 1.37 & 1.94 & 1.15 & 2.5 & std $\%$ \\
\hline
\end{tabular}



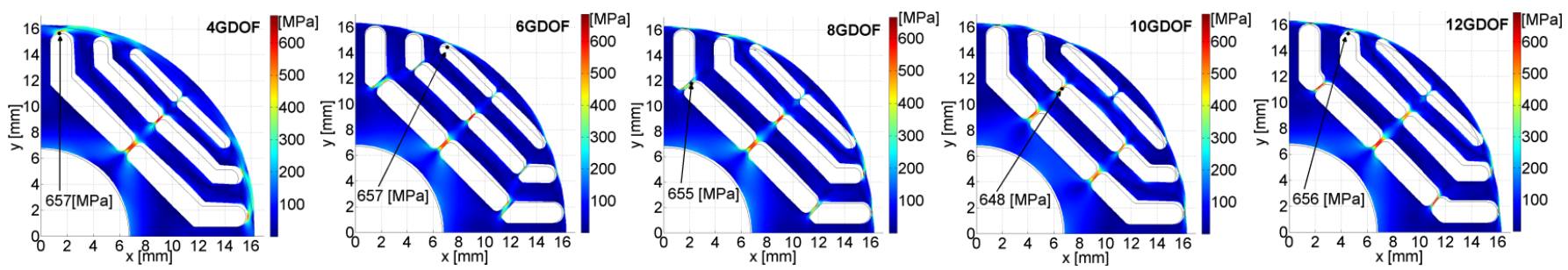

Fig. 5: Von Mises stress distribution of the optimized rotor geometries with a 1:30 scale for the deformation

lateral iron ribs are at $52 \%$ and $65 \%$ of the respective barrier lengths. This distribution achieves the best performance in terms of average total bridge and therefore electromagnetic torque. Comparing rotors showing 10 and 12 GDOF, since they have the same $A T B s$ and so electromagnetic torque, it can be deduced that more than one optimal geometry exists for a given value of average total bridge per flux barrier. This indicates that the solution of the structural optimization problem, as it has been conceived here, for the rotor having 10 and 12 GDOF is not unique. This is also proven by the observation that the optimizations of the rotors with 10 and 12 GDOF are more likely to provide a different final geometry, when compared to the optimizations of the rotors having 4, 6 and $8 G D O F$ as it is shown in Table IV. The latter reports: 1) the standard deviation of the objective function $\operatorname{std} A T B$ calculated over the 10 results obtained for each rotor, 2) the euclidean distances $E D$ (evaluated considering the design variables) between the farthest and the nearest solution with respect to the average and 3 ) the difference $\triangle E D$ between the previous two distances. Even if $\operatorname{std} A T B$ remains quite low for all the 5 sets of optimizations, $\triangle E D$, which clearly represents how much different the obtained results are in terms of geometry, is much bigger for the rotor with 10 and 12 $G D O F$ than the others with less degrees of freedom.

TABLE IV: Standard deviations of the objective functions and euclidean distances among the design variables calculated considering 10 optimization runs for each rotor geometry

\begin{tabular}{cccccc}
\hline & 4 GDOF & 6 GDOF & 8 GDOF & 10 GDOF & 12 GDOF \\
\hline stdATB $(\%)$ & 0.68 & 5.09 & 2.09 & 4.87 & 3.55 \\
$\min E D$ & 0.015 & 0.024 & 0.018 & 0.093 & 0.171 \\
$\max E D$ & 0.082 & 0.097 & 0.106 & 0.5 & 0.565 \\
$\Delta E D$ & $\mathbf{0 . 0 6 7}$ & $\mathbf{0 . 0 7 3}$ & $\mathbf{0 . 0 8 8}$ & $\mathbf{0 . 4 0 7}$ & $\mathbf{0 . 3 9 4}$ \\
\hline
\end{tabular}

\section{Rotor-shaft coupling influence}

The results shown in the last section have been obtained by means of structural optimizations which neglect the influence of the rotor-shaft fitting. This is required to guarantee the transmission of the torque and to avoid that the two assembled parts separate from each other at the maximum operating speed [26]. In this case study, the coupling is assured by the wellknown method of interference fit which of course causes an additional stress at the mating surface (called interference or contact pressure). This pressure negatively affects the rotor von Mises stress distribution and so the maximum stress; its influence becomes significant as the maximum operating speed increases. In fact, the contact pressure depends on the interference, temperature, dimensions and materials properties of the assembled components. In turn the amount of interference, required to avoid the separation of the rotor from the shaft, increases as the maximum speed rises. It is clear that adopting the interference fit, the maximum stress experienced in the rotor will increase, therefore the dimension of the iron bridges have to be redesigned. If such modifications are done manually, the obtained solution might not be optimal anymore. In order to identify the influence on the rotor design due to the interference fit, several structural optimizations have been carried out considering the geometries having 8 and 12 GDOF taking into account the contact pressure. The calculation of the needed interference and the resulting pressure can be done analytically for simple cylindrical parts [26] while it requires a set of FE simulation in case of non-standard geometry. In this study the interference pressure used during the optimizations has been analytically estimated approximating the rotor as a cylindrical component (i.e. without flux barrier) and then verified by FE for one rotor geometry. It has to be underlined that the amount of interference and so the related pressure can lie with a certain range defined by the imposed manufacturing tolerance of the inner rotor bore and outer shaft diameter. The pressure (calculated at the maximum speed) considered during the optimizations (50 $\mathrm{MPa}$ ) corresponds to the worst case scenario in terms of interference. Table $\mathrm{V}$ reports the iron bridge dimensions and the electromagnetic performance of the optimized rotors while Fig. 6 shows their von Mises stress distributions. In this case, increasing the number of $G D O F$ (from 8 to 12 ) leads to an improvement in terms of average total bridge per barrier of about $26 \%$ which implies an increment of the torque of $6 \%$. Obviously the better electromagnetic performance comes at the price of an increased computational effort. Comparing the final geometries obtained with and without considering the contact pressure during the structural optimization, it can be deduced that optimizing also the positions and the bending angles of the lateral iron bridges allows a remarkable advantage. In fact, the average total bridge of the rotor with $8 G D O F$ is incremented of about $35 \%$ while the geometry with $12 G D O F$ requires an $A T B$ increment of only $10 \%$ in order to sustain the interference pressure and keep the maximum stress at the same level. It is important to note that the iron bridge of the optimal rotors obtained considering the contact pressure during the optimization are completely different from the counterpart solutions obtained neglecting the interference pressure. Consequently any attempts to modify the rotor optimized without taking into account the rotor-shaft coupling in order to bring the maximum stress below the limit would most probably lead to a suboptimal solution. 


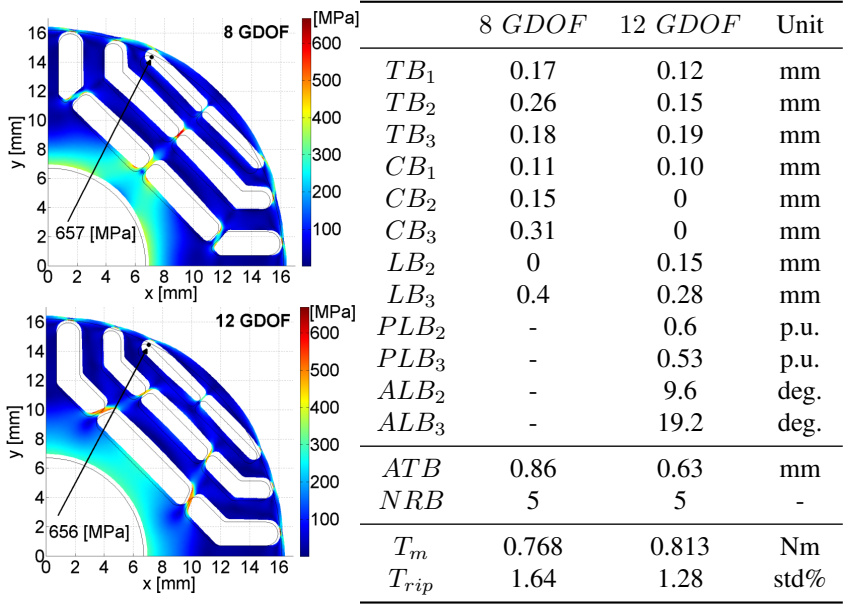

Fig. 6 \& TABLE V: Geometrical parameters, electromagnetic performance and von Mises stress distribution of the rotors optimized considering the interference pressure

\section{Conclusive remarks}

Considering the results of the optimizations carried out neglecting the interference pressure, the following conclusion can be drawn.

- As the geometrical complexity grows, the improvements of the mean torque is remarkable at the beginning (from 4 to 6 $G D O F$ ), then tends to weaken (from 6 to $10 G D O F$ ) until it becomes null for the most complicated rotor structure.

- The best compromise between obtainable performance and required computational time is achieved when adopting the geometry with $6 G D O F$. The latter is obtained optimizing a unique tangential bridge width (common to all barriers), the widths of the lateral and central ribs (which can be different for all barriers), and considering fixed the position and the bending angle of the lateral bridges.

- Adding only the possibility of having different tangential bridge widths (i.e. adopting the $8 G D O F$ rotor) allows reducing the number of radial iron bridges. This can make the rotor manufacturing easier especially in case of small rotor diameters.

- The highest electromagnetic torque is achieved when the positions of the lateral bridges are also included in the structural optimization (i.e. adopting the rotor with 10 $G D O F)$.

- If the positions and the bending angles of the lateral ribs are considered during the structural design, for a given average total bridge corresponds more than one rotor geometry. Consequently the solution of the structural optimization problem is not unique.

When the interference pressure is considered during the structural optimization:

- as $G D O F$ rises, the average total bridge decreases, therefore better performance can be obtained at the cost of an increased computational effort;

- the position and the bending angle of the lateral iron ribs play a fundamental role in the reduction of the maximum stress and so in the minimization of $A T B$.

\section{Simulated PERFORMANCES OF THE FINAL DESIGN}

Considering all the above, then it is clear that the rotor geometry with 12 GDOF (optimized considering the rotorshaft coupling by interference fit) shows the best performance. This machine has therefore been selected and further analysed by means of an accurate FEA. In particular, Fig. 7 shows the average torque, the torque ripple and the power factor as function of the current amplitude at the MTPA conditions. Along the MTPA trajectory the torque ripple remains below $2 \%$ (std, i.e. $7 \%$ calculated considering the usual approach) for current amplitude higher than half of the rated value (21.5 $\left.A_{p k}\right)$. At the rated condition, the optimal machine shows a power factor of 0.53; however its value is quite stable for current amplitude higher than $10 A_{p k}$. Figure 8 depicts the constant loci of the efficiency and the ratio between the copper and the total losses in the torque-speed plane imposing the MTPA as control strategy in the entire speed range. All the electromagnetic loss components have been FE-estimated adopting the approaches presented in [2], [27] respectively for the iron losses and the copper losses. The windage losses occurring in the airgap have been calculated using the semiempirical model presented in [28]. The efficiency is always above $80 \%$ in the region of practical interest (i.e. speed and torque higher than $10 \mathrm{krpm}$ and $0.1 \mathrm{Nm}$ respectively). As the speed increases at the rated torque level, the efficiency tends to sharply increase (between 0-20 krpm), then its rate of increment drastically decreases (between 20-30 krpm) and finally after a stable region $(30-65 \mathrm{krpm})$ slightly tends to decrease. Considering the efficiency along the rated power

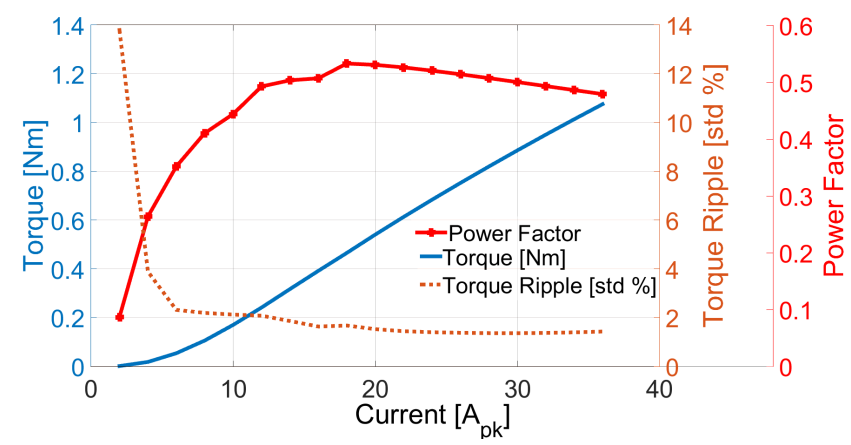

Fig. 7: Average torque, torque ripple and power factor as function of the current amplitude at the MTPA condition
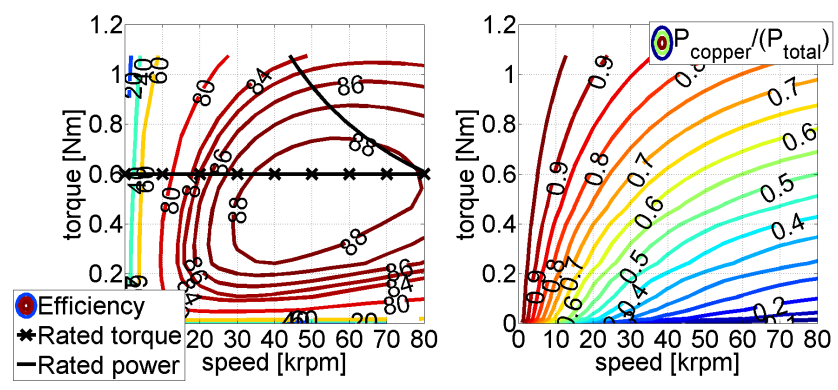

Fig. 8: Efficiency and loss ratio (copper over total losses) constant loci in the torque-speed plane 
locus, as the speed increases the efficiency improves of about $4 \%$. As expected, the relative weight of the copper losses (both $\mathrm{dc}$ and ac components) compared to the total losses decreases as the speed increases and the rate of decrement depends on the torque level as clearly shown in Fig. 8.

\section{EXPERIMENTAL TESTS}

In order to validate all the above considerations, a prototype of the optimized machine has been developed and tested on an instrumented test-rig. To ensure the best possible accuracy, both stator and rotor laminations were cut using the electrical discharge method and these are shown as stacks before the winding and the shaft insertion in Fig. 9. In the following a brief description of the test layout is presented along with the results of the initial experimental tests including the magnetic model identification and the no-load and load dynamic tests.

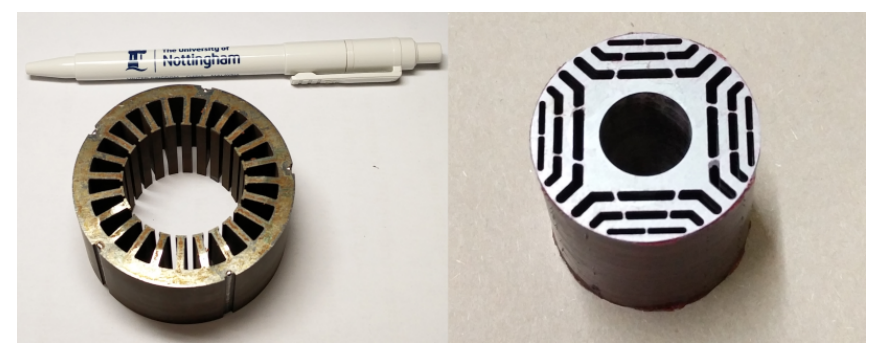

Fig. 9: Stator and rotor lamination stacks before winding and assembling (pictures are not shown in the same scale)

\section{A. Test set-up}

The prototyped HS-SyR machine was coupled to a load motor (a $37 \mathrm{~kW}, 20 \mathrm{krpm}$ IM) via a speed increasing gearbox having ratio 1:5.975 and a $3.5 \mathrm{Nm}$ torque transducer (see Fig. 10). During the test, the load IM was supplied via a 4quadrant regenerative drive, which was set to operate in speed control mode during the magnetic model identification test and in torque control mode during the dynamic tests. The prototyped machine was supplied by an in-house designed three phase full bridge converter featuring $\mathrm{SiC}$ power modules

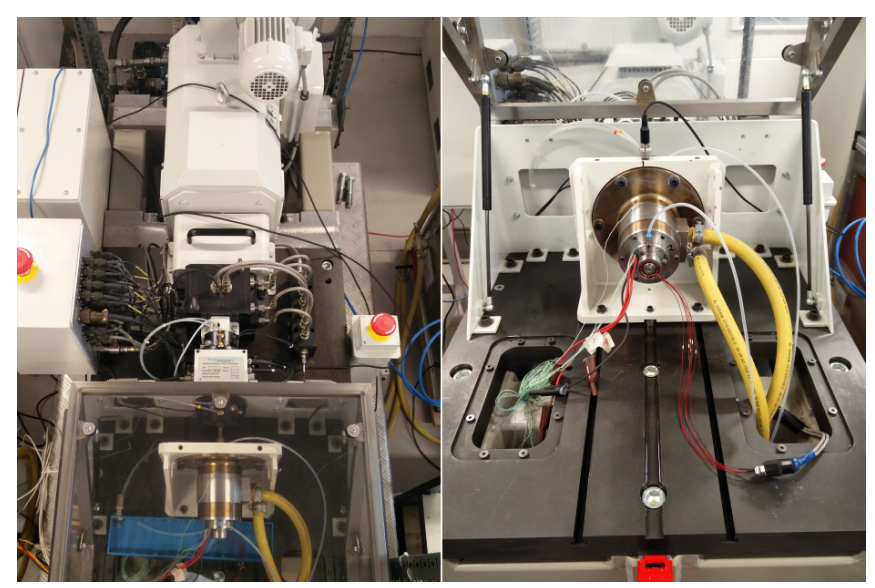

Fig. 10: Layout of the test rig (left), developed machine mounted on the test rig bracket (right) whose full specifications can be found in [29], [30]. The control platform used for acquiring the currents and rotor position measurements, to implement the control algorithm and to generate the control signals for the gate drivers is based on a Xilinx Zynq7020 SoC. During the tests, the switching frequency was set to $40 \mathrm{kHz}$ guaranteeing an appropriate ratio between the switching and the fundamental frequency over the whole operating speed range.

\section{B. Magnetic model identification}

In order to validate the FE-calculated performance and perform an accurate vector control, the magnetic model (i.e. the flux-current relationships of the developed machine) has been identified adopting the procedure presented in [31]. Fig. 11 reports the measured d- and q-axis fluxes (dashed lines) with the FE-calculated ones (continuous lines) as function of both current components. As can be observed, the measured q-axis flux is very similar to the FE-predicted one over the whole d- and q-axis currents range. In particular, the maximum discrepancy between the measured and the FE q-axis flux (about 3\%) is located in the knee region of curve while in the rated condition is about $1 \%$. On the other hand, the d-axis flux comparison presents a much higher discrepancy between measured and predicted results, where the worst case shows the measured flux as $7 \%$ lower than the predicted one in the rated condition. This difference can be ascribed to the variations of the iron bridge dimensions due to the

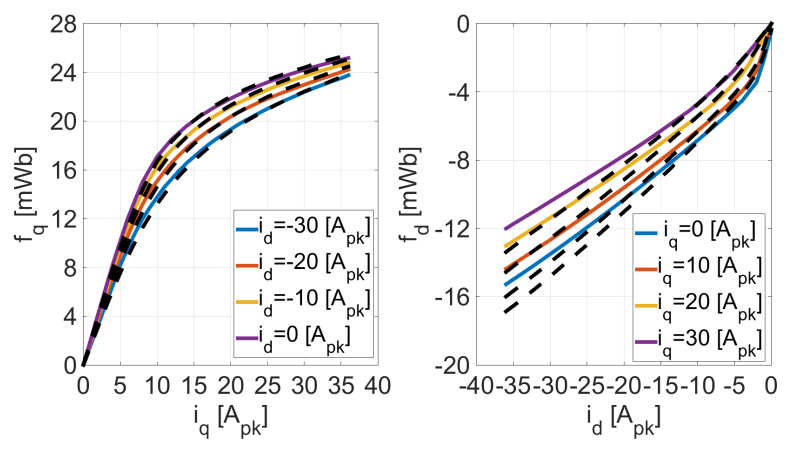

Fig. 11: FE-calculated (continuous lines) and experimental estimated (dashed lines) flux linkages as function of the d- and q-axis currents

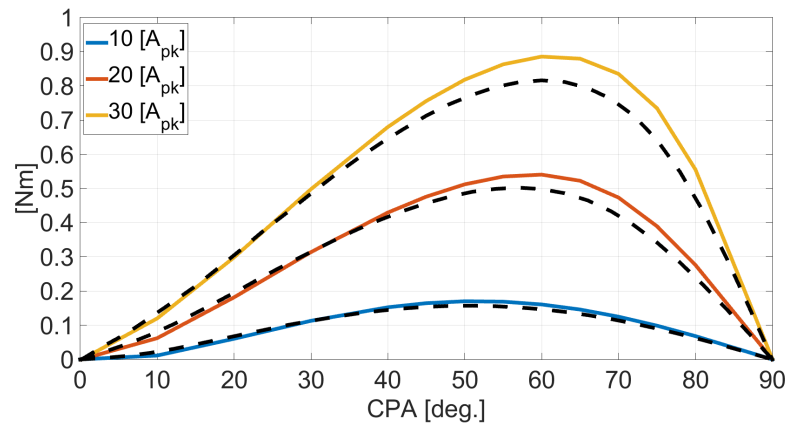

Fig. 12: FE-calculated (continuous lines) and experimental estimated (dashed lines) torque as function of the current phase angle (CPA) and current amplitude 
manufacturing tolerance. The obvious consequence of this dissimilarity between the expected flux and the real one is that the produced torque also shows a discrepancy between measured and predicted results as reported in Fig. 12. The decrement of the torque over the whole range of the current amplitude and phase angle is always below $8 \%$.

\section{Dynamic tests}

The identified magnet model has been used to build the look-up tables expressing the torque and the inductances along the MTPA trajectory needed to calculate the $\mathrm{d}$ - and q-axis current references and the decoupling terms of the standard vector control scheme. The measured inductances have been also used to finely tune the constants of the PI current controllers. After some preliminary tests aimed at verifying the dynamic behaviour of the control system, a motoring noload test has been performed imposing a trapezoidal speed reference reaching a maximum of $35 \mathrm{krpm}$ as shown in Fig. 13. The latter, which reports the measured and the reference speed and d- and q-axis currents, shows the good dynamic behaviour of the control system and the low value of the current and so of the friction losses (i.e. windage and bearing losses). Once the integrity of the built prototype was verified, the machine was coupled with the loading IM in order to carry out a set of motoring load test aimed at measuring the machine efficiency at speeds varying from 6 to $30 \mathrm{krpm}$ and torques from 0.1 to $0.8 \mathrm{Nm}$. For every speed and load, the tests were performed reaching the thermal steady state and acquiring at regular sampling time input and output power

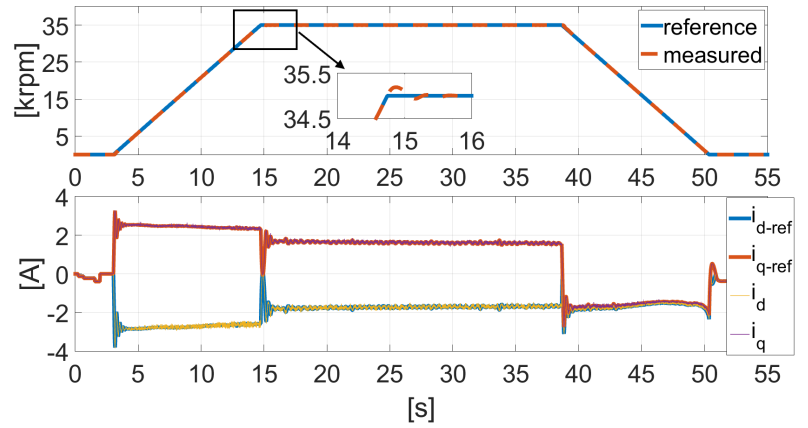

Fig. 13: Measured speed and current transient during a no-load test

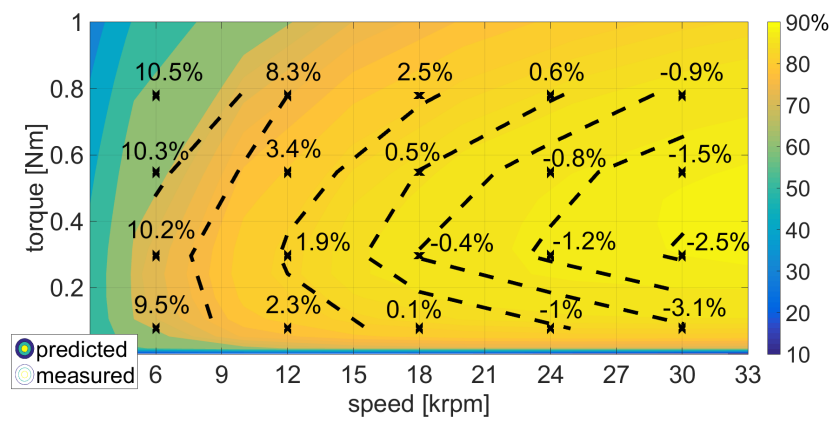

Fig. 14: Contour of the estimated (coloured) and measured (dashed lines) efficiencies in the torque-speed plane and the relative difference between the estimated and the measured efficiency via respectively the torque-meter and the power analyser. Fig. 14 reports the predicted and measured efficiency and the percentage errors between them in the torque-speed plane. The measured efficiency shows essentially the same trends of the estimated ones and their difference is always below 10\%. This discrepancy is obviously due to 1) the lower electromagnetic torque respect to the FE prediction (caused by the influence of the manufacturing tolerances) and 2) the inevitable uncertainties in the evaluation of all loss components.

\section{CONCLUSION}

In this paper a comprehensive study on the rotor design of HS-SyR machines has been given. A two steps design procedure has been proposed and implemented, where first the electromagnetic design is carried out considering a subset of the rotor geometrical parameters then the structural design is performed on the remaining variables. This work has focused on the structural design of such machines, addressed by means of stochastic optimization coupled with structural FEA. It has been shown that the minimization of the average total bridge per flux barrier subject to the constraint on the maximum stress leads to rotor structure with the highest average torque. The comparative optimization study performed on rotor geometries having different degrees of freedom in terms of iron bridge dimensions and distributions has showed how to reach the best trade-offs between the electromagnetic performance, the computational effort and the rotor manufacturability. According to the presented analysis, the rotor geometry showing the best electromagnetic performance has been further analysed by FEA, manufactured and tested on an instrumented test rig. The measured flux-current characteristics are in good agreement with the FE model; the small discrepancy between the measured and the expected performance is due to the rotor manufacturing tolerances. The developed machine was successfully tested up to $30 \mathrm{krpm}$ and $130 \%$ the rated torque showing a measured efficiency of $88 \%$ which matches quite closely the initially estimated $87 \%$. Further test rig modifications have been undertaken to improve the machine-loading rig alignment and address other limitations of the employed housing design in order to allow tests in the higher speed range.

\section{ACKNOWLEDGMENT}

The authors would like to thank Nippon Steel \& Sumitomo Metal Corporation for providing the laminations used for the rotor completely free of charge and Cummins Generator Technologies for the financial support.

\section{REFERENCES}

[1] A. Boglietti, A. Cavagnino, M. Pastorelli, D. Staton, and A. Vagati. Thermal analysis of induction and synchronous reluctance motors. Industry Applications, IEEE Transactions on, 42(3):675-680, 2006.

[2] G. Pellegrino, A. Vagati, B. Boazzo, and P. Guglielmi. Comparison of induction and pm synchronous motor drives for ev application including design examples. Industry Applications, IEEE Transactions on, 48(6):2322-2332, 2012

[3] D.A. Staton, T.J.E. Miller, and S.E. Wood. Maximising the saliency ratio of the synchronous reluctance motor. Electric Power Applications, IEE Proceedings B, 140(4):249-259, July 1993. 
[4] A. Fratta, G. P. Toglia, A. Vagati, and F. Villata. Ripple evaluation of high-performance synchronous reluctance machines. Industry Applications Magazine, IEEE, 1(4):14-22, 1995.

[5] A. Vagati, M. Pastorelli, G. Francheschini, and S. C. Petrache. Design of low-torque-ripple synchronous reluctance motors. Industry Applications, IEEE Transactions on, 34(4):758-765, 1998.

[6] N. Bianchi, M. Degano, and E. Fornasiero. Sensitivity analysis of torque ripple reduction of synchronous reluctance and interior pm motors. Industry Applications, IEEE Transactions on, 51(1):187-195, 2015.

[7] N. Bianchi, E. Fornasiero, and Soong Wen. Selection of pm flux linkage for maximum low-speed torque rating in a pm-assisted synchronous reluctance machine. Industry Applications, IEEE Transactions on, 51(5):3600-3608, 2015.

[8] J. Ikheimo, J. Kolehmainen, T. Knskangas, V. Kivel, and R. R. Moghaddam. Synchronous high-speed reluctance machine with novel rotor construction. IEEE Transactions on Industrial Electronics, 61(6):29692975, June 2014.

[9] P. B. Reddy, K. Grace, and A. El-Refaie. Conceptual design of sleeve rotor synchronous reluctance motor for traction applications. IET Electric Power Applications, 10(5):368-374, 2016.

[10] A. M. EL-Refaie, R. Manzke, and T. M. Jahns. Application of bi-state magnetic material to automotive offset-coupled ipm starter/alternator machine. IEEE Transactions on Industry Applications, 40(3):717-725, May 2004.

[11] M. Palmieri, M. Perta, F. Cupertino, and G. Pellegrino. High-speed scalability of synchronous reluctance machines considering different lamination materials. In Industrial Electronics Society, IECON 2014 - 40th Annual Conference of the IEEE, pages 614-620.

[12] I. Tanaka, H. Nitomi, K. Imanishi, K. Okamura, and H. Yashiki. Application of high-strength nonoriented electrical steel to interior permanent magnet synchronous motor. IEEE Transactions on Magnetics, 49(6):2997-3001, June 2013.

[13] M. Palmieri, M. Perta, and F. Cupertino. Design of a 50.000-r/min synchronous reluctance machine for an aeronautic diesel engine compressor. IEEE Transactions on Industry Applications, 52(5):3831-3838, Sept 2016.

[14] M. Di Nardo, M. Galea, C. Gerada, M. Palmieri, and F. Cupertino. Multiphysics optimization strategies for high speed synchronous reluctance machines. In Energy Conversion Congress and Exposition (ECCE), 2015 IEEE, pages 2813-2820.

[15] M. Di Nardo, M. Galea, C. Gerada, M. Palmieri, F. Cupertino, and S. Mebarki. Comparison of multi-physics optimization methods for high speed synchronous reluctance machines. In Industrial Electronics Society, IECON 2015 - 41st Annual Conference of the IEEE, pages 002771-002776, Nov 2015.

[16] M. Barcaro, G. Meneghetti, and N. Bianchi. Structural analysis of the interior pm rotor considering both static and fatigue loading. Industry Applications, IEEE Transactions on, 50(1):253-260, 2014.

[17] A. Binder, T. Schneider, and M. Klohr. Fixation of buried and surface-mounted magnets in high-speed permanent-magnet synchronous machines. Industry Applications, IEEE Transactions on, 42(4):10311037, July 2006.

[18] A. Vagati, G. Franceschini, I. Marongiu, and G. P. Troglia. Design criteria of high performance synchronous reluctance motors. In Industry Applications Society Annual Meeting, 1992., Conference Record of the 1992 IEEE, pages 66-73 vol.1.

[19] M. Gamba, G. Pellegrino, and A. Vagati. A new pm-assisted synchronous reluctance machine with a nonconventional fractional slot per pole combination. In Optimization of Electrical and Electronic Equipment (OPTIM), 2014 International Conference on, pages 268-275.

[20] R. R. Moghaddam and F. Gyllensten. Novel high-performance synrm design method: An easy approach for a complicated rotor topology. Industrial Electronics, IEEE Transactions on, 61(9):5058-5065, 2014.

[21] M. Gamba, G. Pellegrino, and F. Cupertino. Optimal number of rotor parameters for the automatic design of synchronous reluctance machines. In Electrical Machines (ICEM), 2014 International Conference on, pages $1334-1340$

[22] M. Di Nardo, M. Degano, M. Galea, C. Gerada, M. Palmieri, F. Cupertino, N. Bianchi, and D. Gerada. End barrier shape optimizations and sensitivity analysis of synchronous reluctance machines. In Industrial Electronics Society, IECON 2015 - 41st Annual Conference of the IEEE, pages 002914-002919, Nov 2015.

[23] A. Tessarolo. Modeling and analysis of synchronous reluctance machines with circular flux barriers through conformal mapping. Magnetics, IEEE Transactions on, 51(4):1-11, April 2015.

[24] H. Mahmoud and N. Bianchi. Non-linear analytical model of eccentric synchronous reluctance machines considering the iron saturation and slotting effect. IEEE Transactions on Industry Applications, $\mathrm{PP}(99): 1-$ $1,2017$.

[25] M. Di Nardo, G. L. Calzo, M. Galea, and C. Gerada. Structural design optimization of a high speed synchronous reluctance machine. In 2016 XXII International Conference on Electrical Machines (ICEM), pages 2073-2079, Sept 2016.

[26] Wei Tong. Mechanical Design of Electrical Motors.

[27] M. van der Geest, H. Polinder, J. A. Ferreira, and D. Zeilstra. Current sharing analysis of parallel strands in low-voltage high-speed machines. IEEE Transactions on Industrial Electronics, 61(6):3064-3070, June 2014.

[28] J. Nerg, M. Rilla, and J. Pyrhonen. Thermal analysis of radial-flux electrical machines with a high power density. IEEE Transactions on Industrial Electronics, 55(10):3543-3554, Oct 2008.

[29] G. L. Calzo, P. Zanchetta, C. Gerada, A. Gaeta, and F. Crescimbini. Converter topologies comparison for more electric aircrafts high speed starter/generator application. In 2015 IEEE Energy Conversion Congress and Exposition (ECCE), pages 3659-3666, Sept 2015.

[30] Giovanni Lo Calzo. Power generating unit for the future more electric aircraft : power electronic converter modelling and design. $\mathrm{PhD}$ thesis, Universita' degli studi Roma Tre, 2015.

[31] E. Armando, R. I. Bojoi, P. Guglielmi, G. Pellegrino, and M. Pastorelli. Experimental identification of the magnetic model of synchronous machines. Industry Applications, IEEE Transactions on, 49(5):21162125,2013 